\title{
Risk Tolerance and Social Awareness: Adapting Deterrence Sanctions to Agent Populations
}

\author{
Henrique Lopes Cardoso and Eugénio Oliveira \\ LIACC, DEI / Faculdade de Engenharia, Universidade do Porto \\ R. Dr. Roberto Frias, 4200-465 Porto, Portugal \\ hlc@fe.up.pt, eco@fe.up.pt
}

\begin{abstract}
Normative environments for multi-agent systems provide means to monitor and enforce agents' compliance to their commitments. However, when the normative space is imperfect, contracts to which norms apply may be unbalanced, and agents may exploit potential flaws to their own advantage. In this paper we analyze how a normative framework endowed with a simple adaptive deterrence sanctioning model responds to different agent populations. Agents are characterized by their risk tolerance and by their social attitude. We show that risk-averse or socially concerned populations cause lesser deterrence sanctions to be imposed by the normative system.
\end{abstract}

\section{Introduction}

Interaction infrastructures for multi-agent systems have been extensively studied. Within such efforts, some attention has been given to normative environments (e.g. [1-3]), consisting of middleware that provides support for explicitly handling normative relations among agents (such as contracts specified through normative operators, e.g. obligations). Fewer efforts have been put on the development of dynamic capabilities in normative environments, where the infrastructure itself seeks to adapt its enforcement policies to the actual interaction scenario that is taking place.

In fact, when embedded in some notion of "institution", normative environments take an active role in checking agents' compliance with their commitments, and furthermore in enforcing such compliance. The goal is to establish trust among participants in a norm-regulated relationship - this gives contracts a binding force.

An important feature of interaction infrastructures with a contracting emphasis is the assistance of contract formation, namely by providing a normative framework that specifies norms applicable to different contractual settings. Given that complete contract negotiation automation is not likely to be possible (both in terms of technological limitations and real-world acceptance), software agents may rely on background normative frameworks that fill-in the normative body of contracts. This feature is particularly important when considering contrary-to-duty situations, which should describe a normative response in case of non-compliance to contractual terms. In certain cases, however, there will be no specified response. This is where other coercive approaches may be relevant, in situations where agents try to take advantage of their potential gain when violating norms (because they might be more self-interested than socially concerned). 
In the literature (e.g. $[4,5])$ we find two basic kinds of sanctions that an institution may apply in order to incentive norm compliance (or, to put it another way, to discourage deviations). Direct material sanctions have an immediate effect, and consist of affecting the resources an agent has (e.g. by applying fines). Indirect social sanctions, such as changing an agent's reputation, may have an effect that extends through time. Depending on the domain and on the set of agents that inhabit the institutional environment, the effectiveness of such sanctions may be different: if agents are not able to take advantage of other agents' reputation information, material sanctions should be used instead.

There are two general policies used when applying (direct) sanctions, which concern their intended effects: (i) deterrence aims at punishing the violator so as to discourage future violations; (ii) retribution aims at compensating the addressee of the violation. Bringing these policies to an electronic institution realm, we consider that retribution sanctions are those specified in contractual norms, be they negotiated or inherited from a preexistent normative framework. In this case the institution, while monitoring norm compliance, acts as a mediator. As for deterrence sanctions, they will be applied by the institution itself, and may be used so as to maintain order (by motivating agents to comply) and consequently trust in the system.

Deterrence has been studied in political science [6] with a different perspective, where theories are proposed for explaining international relations in tense periods such as the Cold War. Deterrence is based on threats between different nations.

Economic approaches to law enforcement have suggested analyzing sanctions and their amplitude by taking into account their effects on parties' activities. Agents committing to norms that have associated deterrence sanctions enter risky activities, because they may unintentionally violate them. It has been argued [7] that under strict liability (where violators are always sanctioned) sanctions should equal harm done. An increase in the level of activity brings an increase in the expected harm; if damages imposed by sanctions equal harm, parties will have socially correct incentives to engage in risky activities (that is, to establish commitments). However, this conclusion relies on the additional assumption that parties are risk-neutral. According to [7], if agents are risk-averse the optimal level of damages tends to be lower than harm. With risk-aversion, a sanction imposes a cost which does not exist under risk neutrality: risk-aversion introduces costless deterrence [8] and the policy-maker should take that into account when choosing the optimal sanction.

Our normative environment model makes two simplifying assumptions: 1) strict liability (norm violations are always detected); 2) costless enforcement (monitoring and sanctioning have a negligible cost to the institution).

With these assumptions in place, we will describe an adaptive model for a normative framework. The model tries to avoid over-constraining the environment while ensuring a certain level of norm compliance, by adjusting deterrence sanctions.

In this paper we analyze how the adaptive model responds to different agent populations, where each agent is characterized by a risk tolerance parameter and a social awareness parameter. The former represents an agent's willingness to contract in the presence of violation fines. The latter allows us to embed in our agents some notion of social welfare; that is, although we will take agents as utility maximizers, agents are not all equally self-interested. 
The rest of the paper is organized as follows. Section 2 introduces the reader with the adaptive model for deterrence sanctions, and Section 3 provides a feeling of how the model works with a simple scenario. Section 4 presents several experiments conducted with various agent populations, with different risk tolerance and social awareness distributions. Finally, Section 5 concludes.

\section{A Normative Framework with Deterrence Sanctions}

In our approach we take the stance that agents are truly autonomous, and thus cannot be forced to fulfill their obligations. The institution may, however, impose certain fines as deterrence sanctions: such fines are assumed to be fully regimented (that is, agents cannot escape them, e.g. because they were required, upon entering the institution, to make a deposit that is in control of the institution).

We are mainly concerned with contracting scenarios, in which agents make mutual commitments and create business expectations. Violations, even when handled by contractual norms, are seen as abnormal situations. Thus, if a certain kind of violation becomes frequent, response should be taken through an increase of sanctions.

\subsection{Commitment Trees}

In order to obtain a tractable model for handling contractual commitments, we use a tree-based representation for interdependent obligations. This representation is useful for understanding the simulation model that we use.

When establishing contracts, agents create a network of directed obligations, some of which are dependent on the fulfillment or violation of other obligations. Consider the following two-party contract: agent $a$ will pay $p$ units to agent $b$, after which $b$ will deliver $x$ to $a$. In case $b$ fails to deliver, he must return $p^{\prime}=p+\delta$ to $a$. This sequence of commitments is illustrated in Figure 1, in a tree-like structure - a commitment tree ${ }^{1}$. Each node represents an obligation, and each labeled directed edge indicates, in the child node pointed to, what follows when the obligation contained in the parent node is fulfilled (Fulf) or violated ( Viol). Note that in this simple example nothing is specified yet if agent $a$ violates his commitment to pay $p$, nor if agent $b$ violates his commitment to return $p^{\prime}$.

The violation of an obligation with a prescribed sanction may simply denote a case where an agent preferred to incur the sanctions for matters of conflicting goals (e.g. he had another contract which was more important than this one, and could not stand for both). If such violation becomes frequent, however, this may denote a flaw in the normative system that agents are being able to exploit to their own advantage.

The importance of adaptation in a normative framework resides in the fact that contracts may be unfair in certain execution outcomes. If self-interested agents try to explore such flaws to their own profit, action should be taken so as to discourage such behaviors. Aiming at a model that adapts the normative framework in a domainindependent way, we focus on adding deterrence fines to the system (which are not violable), instead of adjusting prescribed obligations in each violation situation.

\footnotetext{
${ }^{1}$ Although the commitment structure may be more complex, we simplify it to a binary tree; while not limiting the applicability of our adaptation approach, this choice makes the model easier to follow.
} 


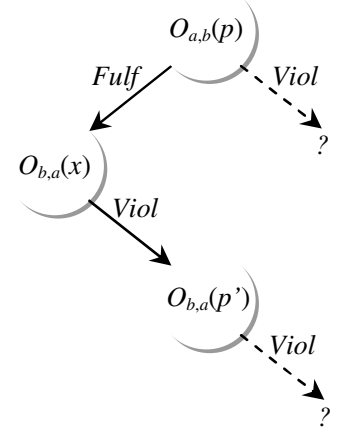

Fig. 1. Sample commitment tree

The normative framework's adaptation is based on associating, with each obligation, a fine that can be strengthened or weakened. With this approach, every obligation will have a (potentially null) fine to be imposed on the bearer in case of violation; this fine is added up to the violation consequence in the child node already in the tree, if there is one. The fine-update function is based on fine application frequency. Fines will increase when they are applied often, and decrease when they are not used. A low level of fine usage indicates that obligations are being fulfilled or they are not being used as often as desired: in both cases fines should be decreased, since they either are not needed or are inhibiting activity. On the other hand, a high level of fine usage means that agents still prefer to go through the sanction, and as such it should be increased as a deterrence mechanism. In sum, this approach tries to make fines (a) strong enough to discourage deviation and (b) weak enough to avoid unnecessary institutional control.

\subsection{Contract Enactment}

We developed a simulation prototype that allows us to experiment with the adaptation model briefly introduced above. In our system we shall have a number of agents that will each be given an opportunity to sign a contract. The contract structure is defined by a number of enacting roles and by an underlying binary commitment tree (BCT from now on). Roles are used as bearers or counterparties of the obligations in the tree. Furthermore, each obligation has an associated cost (to be supported by a fulfilling bearer) and benefit (to be collected by the counterparty of a fulfilled obligation). Figure 2 more clearly depicts the characterization of each node in a BCT.

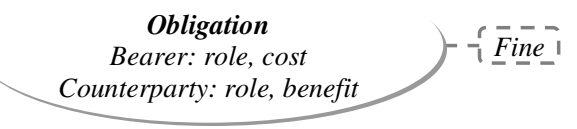

Fig. 2. A BCT node and its configuration 
When an agent decides to sign a contract, he will enact the corresponding commitment tree with a role assigned to him before contracting. We say that the state of a contract enactment is the commitment currently under appreciation. If the bearer of such a commitment is the agent that decided to contract, he will be asked for a play: either to fulfill or to violate such a commitment. If the commitment's bearer is not the agent, the system will decide whether the commitment will be fulfilled or not, according to a uniform strategy. The current state will be updated according to the decision taken: if the choice is to fulfill, the state will become the root commitment of the fulfillment sub-tree; if the choice is to violate, the state will become the root commitment of the violation sub-tree. The contract terminates when the state reaches a null value (no fulfillment/violation sub-tree exists upon a fulfill/violate decision).

\subsection{Agent Decision-Making}

Each agent has two distinct kinds of decisions to make. If he does not have an ongoing contract, he is given the opportunity to sign one. For that, a random role from the contract structure is selected and the agent is asked if he wants to contract with that role. Each agent is configured with a risk tolerance parameter $R t \in[0 ; 1[$, which denotes his willingness to contract in the presence of violation fines. If $R t=0$, the agent decides to contract only if he will be subject to no fines at all. If $R t \approx 1$, the agent will always risk to contract, regardless of any fines. An agent will decide to contract depending on the highest fine that is associated with commitments for the assigned role. In order to contract, the following relation should be true:

$$
\text { highestFine }(\text { role }) \leq b * R t /(1-R t)
$$

where $b$ is a slope parameter associated with the agent's budget. We assume that agents always prefer to contract, regardless of commitment costs or benefits. The contract is presumably beneficial to all partners should they fulfill their commitments.

When an agent has an ongoing contract, if the current state is a commitment with him as the bearer he will decide whether to fulfill or to violate. Depending on a socalled in-contract strategy, the agent will explore the contract's BCT in order to decide which option is best for him. Such strategies may vary from simply comparing the cost of fulfillment with the applicable fine in case of violation, to computing the path with the best outcome from the whole BCT (more on this in Section 3.2).

Agents are essentially expected utility maximizers. This means that, in principle, they will fulfill obligations only when the expected outcome from this choice is better than the expected outcome from violating (according to the employed in-contract strategy). We do however embed in our agents some notion of social welfare, which impels them to fulfill even when they do not have a strict advantage in doing so. While for now we do not consider the effect of reputation in future contracts, we allow in our model that agents are not all equally self-interested. For that we introduce a social awareness parameter $S a \in[0 ; 1$ [. If $S a=0$, the agent will violate whenever the outcome from this choice is better than the outcome from fulfilling. On the other extreme, if $S a \approx 1$ the agent will always choose to fulfill. The agent will decide to fulfill an obligation $o$ whenever the following relation is true:

$$
\text { violationOutcome }(o) \text { - fulfillmentOutcome }(o) \leq b * S a /(1-S a)
$$


where $b$ is a slope parameter associated with the agent's budget. The violation and fulfillment outcomes are calculated by the in-contract strategy.

\subsection{Fine-Update Policy}

After all agents running in the simulation have a chance to play, the contract structure will adapt, that is, fines associated to the BCT will be updated. Each fine is updated independently of all other fines.

In order to delineate a fine update policy, we first need to define the goal function that will be pursuit. As mentioned before, fine updates should take into account how often they are applied. We define a threshold parameter $T h \in[0 ; 1]$ that indicates the highest percentage of fines that the system should accept as normal. For instance, with a value $T h=0.1$ we are saying that if more than $10 \%$ of the agents running in the simulation violate a given obligation, the normative system will raise the fine in the next step - we say that $10 \%$ of the total number of agents is the number of tolerated violations. Furthermore, since not all agents will be in the same state at a given time point, we adjust the threshold according to the number of agents that did in fact make a decision concerning the fulfillment or violation of a specific obligation (because they were in that state). A state's fine will be increased if the number of violations exceeds the following tolerated violations function:

$$
\text { toleratedViolations }=2 * T h * N a g /\left(1+e^{-(5 / N a g) * x}\right)-T h * N a g
$$

where Nag is the number of agents running in the simulation and $x$ is the number of agents that were in this state. This is a sigmoid function with an upper bound set at $T h * N a g$ (a percentage of the total number of agents).

The system should keep fines as low as possible, while still conforming to the goal function outlined above. This is because the overall goal of the system is to maximize contract activity, which should be obtained with less risk exposure in an agent population with unknown risk tolerance. Therefore, whenever the number of violations does not exceed the number of tolerated violations, the fine will be decreased. Fines are increased heavier than they are decreased. We have set an increase step of 0.1 and a decrease step of 0.01 . Fines will be applied rounded to the first decimal place, which gives a sense that it takes ten simulation steps (without exceeding the tolerated violations function) to decrease the fine value.

\section{Scenario}

In this section we present a scenario on which experiences reported in Section 4 are based. We show the deterrence sanction adaptation in a uniform distribution setting.

\subsection{Contract Structure}

Since we are not concerned with the correctness of the contract to be signed, we may experiment with a large number of different BCTs (some arbitrary examples can be found in [9]). However, for space limitations we will concentrate on a simple contract structure with two roles, whose BCT is illustrated in Figure 3. This BCT includes two complementary obligations 0 and 1, and their respective contrary-to-duties 3 and 2 . We shall call obligation 1 the to-duty obligation of obligation 0 . 


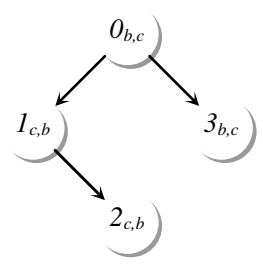

Fig. 3. Binary commitment tree for a contract structure with two roles: each node $I d_{i, j}$ is an obligation where $i$ is the bearer and $j$ is the counterparty. This BCT includes two complementary obligations ( 0 and 1 ) and their contrary-to-duties ( 3 and 2 ).

For all experiments reported in this paper, obligation costs are set at 10.0 and benefits at 12.0 (setting benefits higher than costs tries to give all partners some gain when the contract is well-balanced). Also, fines are initialized at 0.0 .

\subsection{Agent In-Contract Strategy}

As explained in Section 2.3, when deciding whether to comply with commitments agents use an in-contract strategy in order to compute the violation/fulfillment outcomes. Several in-contract strategies can be devised (see [9] for some examples), representing the reasoning abilities of agents when deciding whether to fulfill or violate an obligation.

In this paper we will assume that agents are capable of analyzing the whole BCT, in order to compute the path that will bring them the best possible outcome when assuming that the contract partner will use the same strategy. This is a minimax strategy: the agent will maximize his own expected utility while assuming that the other agent will do the same. For instance, considering the BCT at Figure 3 with no fines, the agent will choose to violate on every obligation. While this seems obvious for obligations 1, 2 and 3 (there is no personal benefit in fulfilling), in obligation 0 the agent chooses to violate because he assumes that the counterparty will violate on 1 and 2, bringing him no benefit that can compensate the cost of fulfilling on 0 .

This strategy seems counterintuitive with the very decision of establishing a contract. However, for the sake of testing the adaptation capabilities of the normative framework, this agent decision practice is bearable.

For all experiments reported in this paper, a uniform strategy "always fulfill" is used by the system for commitments whose bearer is not a simulation agent.

\subsection{Adaptation to Population with Uniform Distribution}

When addressing an agent population with uniform distributions over risk tolerance $(R t)$ and social awareness $(\mathrm{Sa})$ parameters, the adaptation of fines proceeds as illustrated in Figure 4. Every agent will start violating every obligation, which causes an increase of every fine. However, when fines 3 and 2 are high enough, fines 0 and 1 are no longer necessary to persuade agents to fulfill the respective obligations.

We should emphasize that the system tries continuously to lower fines, which is observable by the slight fluctuations of fines towards the end of the curves in figure 4 . Therefore, system imposed fine levels are the lowest that keep violations below the tolerated violations function. 


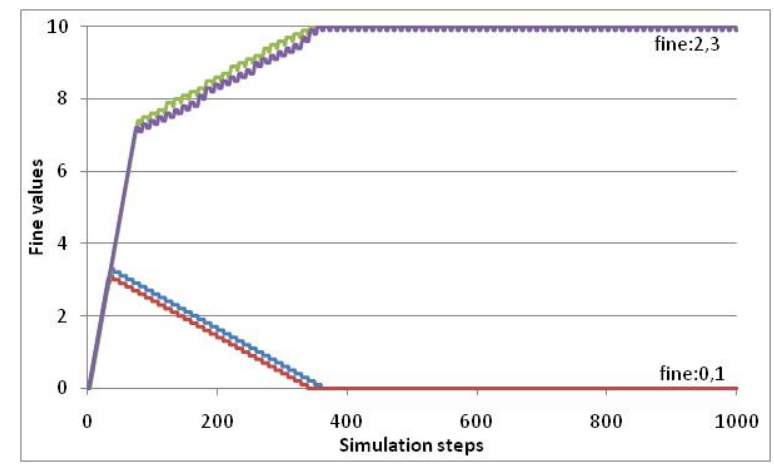

Fig. 4. Fine evolution $(T h=0.1, N a g=10000)$ for 1000 simulation steps with uniform distributions of risk tolerance and social awareness

\section{Adaptation to Different Agent Populations}

In this section we analyze the adaptation of the system when handling different agent populations, in which risk tolerance and social awareness distributions are concerned.

\subsection{Risk Tolerance}

With this first group of experiments we aimed at observing the behavior of the deterrence sanction adaptation model when facing agent populations with different risk

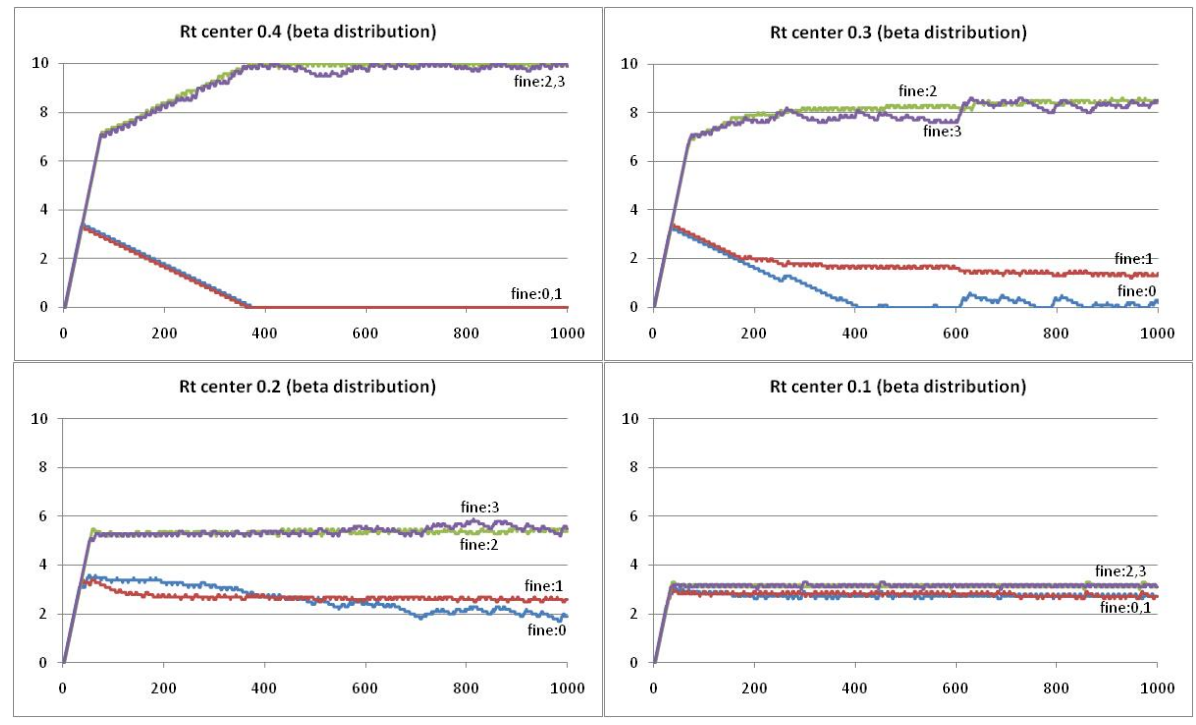

Fig. 5. Fine evolution $(T h=0.1, N a g=10000)$ for 1000 simulation steps with different beta distributions of risk tolerance and uniform distributions of social awareness 
tolerance distributions. In a population that tends to be more risk-averse, higher fines should tend to decrease. In these experiments we used beta distributions centered at different risk tolerance values, in order to represent populations having a predominance of agents with specific risk tolerances. For each beta distribution, we set $\alpha=1+\left(c^{*} p-c\right)$ and $\beta=p-\left(c^{*} p-c\right)$, where $c$ is the center value and $p$ is a peak factor that we have set to 100 .

Figure 5 shows fine evolutions for different risk tolerance center values. As expected, higher fines tend to decrease with lower risk tolerance values. This is due to the fact that, when deciding whether to contract or not, agents compare risk tolerance with the highest applicable fine.

Another interesting observation is that while the highest fines tend to decrease, the system tries to compensate this potentially lower ability to ascertain the desired level of compliance by increasing other sanctions. More specifically, since fines 3 and 2 are lowered, they lose their effect on decisions taken in states 0 and 1 , respectively. As a consequence, fines in these states are raised.

This outcome turns out to be an important emergent property of the normative system: the ability to grasp interdependencies between fines applied to different nodes in the $\mathrm{BCT}$, without being preprogrammed to do so (the fine update policy adapts fines in an independent way). Furthermore, such interdependencies are caused by the incontract strategy used by agents; if agents do not take into account possible "future" fines when making a decision (see [9]), then the system behavior will not pointlessly make a connection between fines.

\subsection{Social Awareness}

With this second group of experiments we aimed at observing the behavior of the deterrence sanction adaptation model when facing agent populations with different social awareness distributions. In a population that tends to be more socially concerned, fines should tend to decrease. Selfish agents will only fulfill if it is in their own interest, while higher social awareness impels agents to fulfill even when they do not benefit directly from that option.

Figure 6 shows fine evolutions for different social awareness center values (using beta distributions as before). As expected, fines tend to increase with lower social awareness values. By doing so, the system tries to discourage commitment violations.
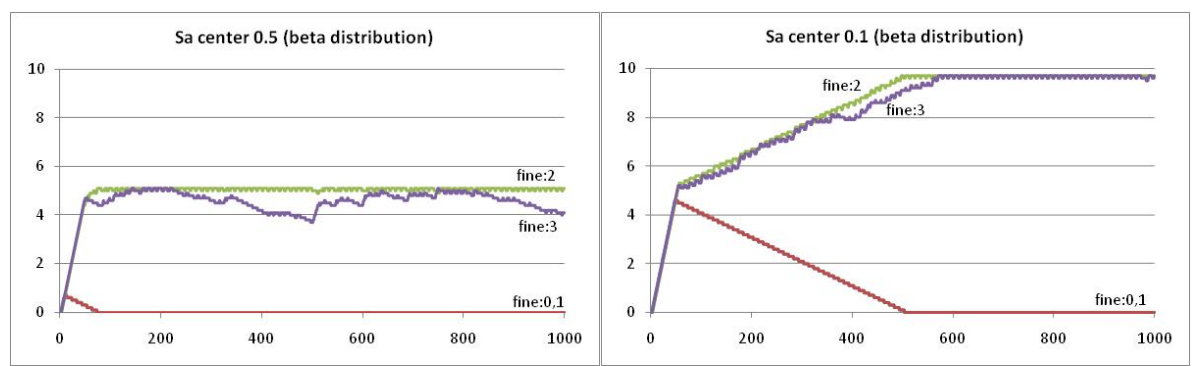

Fig. 6. Fine evolution $(T h=0.1, N a g=10000)$ for 1000 simulation steps with uniform distributions of risk tolerance and different beta distributions of social awareness 
The dependency mentioned before between fines is also visible here: fines 3 and 2 tend to absorb the effects of fines 0 and 1 sooner for higher social awareness values, and the system is able to find these intricacies.

\subsection{Combining Risk Tolerance and Social Awareness}

By adjusting both parameters when setting up an agent population, we get a combination of the effects identified above. Figure 7 shows what happens when we set both risk tolerance and social awareness to beta distributions centered at 0.1 . In this case, since highest fines are limited by a low risk tolerance, the system raises fines 0 and 1 as much as it can, in order to try to force a population of mostly self-interested and risk-averse agents to contract and also comply with contractual commitments. We should add that in these extreme and unlikely conditions the normative system is not successful: the obtained fine levels are insufficient to force compliance, and at the same time too demanding to motivate contractual activity. This means that the few agents that do contract (which nevertheless are in essence risk-averse) will violate their commitments (because they are also too self-interested).

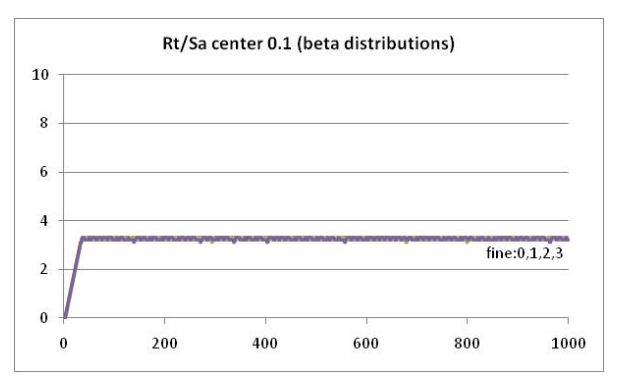

Fig. 7. Fine evolution $(T h=0.1, N a g=10000)$ for 1000 simulation steps with beta distributions of risk tolerance and social awareness centered at 0.1

\section{Conclusions}

Embedding adaptive enforcement mechanisms in normative frameworks is important in open environments. Adapting deterrence levels to the behavior of an agent population is important when the normative space has imperfections that make the contracts to which norms apply unfair, opening the possibility for self-interested agents to exploit their potential advantage.

In this paper we have studied how an adaptive deterrence sanction model that tries to "maintain order" responds when facing different agent populations. Such populations were characterized by a predominant level of risk tolerance and social awareness. The former is inspired on economic theory on deterrence sanctions [7], stating that agents incur a risk when making contracts that are subject to deterrence sanctions. The latter is comparable to other approaches in MAS research that try to model different social attitudes (e.g. [2, 10]).

With the adaptive model described in this paper, imposed fines tend to be lower when agents are more risk-averse or when agents are more socially concerned. Also, 
we observed that when a combination of sanctions is able to drive agents to comply with their commitments, the adaptive mechanism is able to pursue such a combination when constraints limit some options - such constraints are rooted in the agent population (namely the predominant risk tolerance), and are implicitly captured in the fine update policy. This ability is an interesting emergent property of the system.

Dynamic properties of normative systems have been studied from different perspectives. In [11] norms are seen as patterns of behavior that may emerge from agent interactions. In our case, the normative system is external to the agents; we seek to adapt it to a specific agent population in order to pursue an overall system goal.

Sanction-based self-adaptation of institutional normative environments is also studied in [12]. However, their adaptation model is based on the definition of domaindependent transition functions, stating what specific change should be made in a specific norm when some goal specification is not met. Also, their model does not assume strict liability: agents are able to violate norms while not being detected.

In this paper we have not considered the influence of reputation on agent's contractual behavior. It has been argued [13] that in the presence of reputation mechanisms there is a lesser need for deterrence policies. We leave for future work such analysis.

Acknowledgments. The first author is supported by FCT (Fundação para a Ciência e a Tecnologia) under grant SFRH/BD/29773/2006.

\section{References}

1. Arcos, J.L., et al.: Environment engineering for multiagent systems. Engineering Applications of Artificial Intelligence 18(2), 191-204 (2005)

2. Boella, G., van der Torre, L.: A Game-Theoretic Approach to Normative Multi-Agent Systems. In: Normative Multi-agent Systems, Schloss Dagstuhl (2007)

3. Lopes Cardoso, H., Oliveira, E.: A Context-based Institutional Normative Environment. In: Hubner, J., et al. (eds.) Coordination, Organizations, Institutions, and Norms in Agent Systems IV, pp. 140-155. Springer, Heidelberg (2009)

4. Pasquier, P., Flores, R.A., Chaib-Draa, B.: Modelling Flexible Social Commitments and their Enforcement. In: Gleizes, M.-P., Omicini, A., Zambonelli, F. (eds.) Engineering Societies in the Agents World V, pp. 139-151. Springer, Toulouse (2005)

5. Grossi, D., Aldewereld, H., Dignum, F.: Ubi lex, ibi poena: Designing norm enforcement in e-institutions. In: Noriega, P., et al. (eds.) Coordination, Organizations, Institutions, and Norms in Agent Systems II, pp. 101-114. Springer, Heidelberg (2007)

6. Zagare, F.C., Kilgour, D.M.: Perfect Deterrence. Cambridge University Press, Cambridge (2000)

7. Polinsky, A.M., Shavell, S.: Punitive Damages. In: Newman, P. (ed.) The New Palgrave Dictionary of Economics and the Law, pp. 192-198. Stockton Press, New York (1998)

8. Garoupa, N.: The Theory of Optimal Law Enforcement. Journal of Economic Surveys 11(3), 267-295 (1997)

9. Lopes Cardoso, H., Oliveira, E.: Adaptive Deterrence Sanctions in a Normative Framework. In: The 2009 IEEE/WIC/ACM International Conference on Intelligent Agent Technology (IAT 2009). IEEE Computer Society Press, Milan (to appear, 2009) 
10. Castelfranchi, C.: Engineering Social Order. In: Omicini, A., Tolksdorf, R., Zambonelli, F. (eds.) Engineering Societies in the Agents World, pp. 1-18. Springer, Berlin (2000)

11. Sen, S., Airiau, S.: Emergence of norms through social learning. In: 20th Int. J. Conf. on Artificial Intelligence, Hyderabad, India, pp. 1507-1512 (2007)

12. Campos, J., et al.: Formalising Situatedness and Adaptation in Electronic Institutions. In: Hubner, J., et al. (eds.) Coordination, Organizations, Institutions, and Norms in Agent Systems IV, pp. 126-139. Springer, Heidelberg (2009)

13. Luck, M., et al.: Trust and Norms for Interaction. In: Proceedings of the IEEE SMC 2004 International Conference on Systems, Man \& Cybernetics, pp. 1944-1949. IEEE, The Hague (2004) 